\title{
Comparison of a One and Two Parameter Family of Transmission Conditions for Maxwell's Equations with Damping
}

\author{
M. El Bouajaji ${ }^{1}$, V. Dolean ${ }^{2}$, M. J. Gander ${ }^{3}$ and S. Lanteri ${ }^{1}$
}

\section{Introduction}

Transmission conditions between subdomains have a substantial influence on the convergence of iterative domain decomposition algorithms. For Maxwell's equations, transmission conditions which lead to rapidly converging algorithms have been developed both for the curl-curl formulation of Maxwell's equation, see $[2,3,1]$, and also for first order formulations, see [7, 6]. These methods have well found their way into applications, see for example [9] and the references therein. It turns out that good transmission conditions are approximations of transparent boundary conditions. For each form of approximation chosen, one can try to find the best remaining free parameters in the approximation by solving a min-max problem. Usually allowing more free parameters leads to a substantially better solution of the min-max problem, and thus to a much better algorithm. For a particular one parameter family of transmission conditions analyzed in [4], we investigate in this paper a two parameter counterpart. The analysis, which is substantially more complicated than in the one parameter case, reveals that in one particular asymptotic regime there is only negligible improvement possible using two parameters, compared to the one parameter results. This analysis settles an important open question for this family of transmission conditions, and also suggests a direction for systematically reducing the number of parameters in other optimized transmission conditions.

NACHOS project-team, INRIA Sophia Antipolis - Méditerranée research center, F-06902 Sophia Antipolis Cedex, France Mohamed.El_bouajaji@inria.fr, Stephane.Lanteri@inria.fr. Laboratoire J.A. Dieudonné, CNRS UMR 6621, F-06108 Nice Cedex, France dolean@unice.fr · Mathematics Section, University of Geneva, CH-1211, Geneva, Switzerland martin.gander@unige.ch 


\section{Schwarz Methods for Maxwell's Equations}

We consider in this paper a boundary value problem associated to three timeharmonic Maxwell equations with an impedance condition on the boundary of the computational domain $\Omega$,

$$
\begin{aligned}
& -i \omega \varepsilon \mathbf{E}+\operatorname{curl} \mathbf{H}-\sigma \mathbf{E}=\mathbf{J}, i \omega \mu \mathbf{H}+\operatorname{curl} \mathbf{E}=\mathbf{0}, \Omega \\
& \mathscr{B}_{\mathbf{n}}(\mathbf{E}, \mathbf{H}):=\mathbf{n} \times \frac{\mathbf{E}}{Z}+\mathbf{n} \times(\mathbf{H} \times \mathbf{n})=\mathbf{s}, \partial \Omega .
\end{aligned}
$$

with $\mathbf{E}, \mathbf{H}$ being the unknown electric and magnetic fields and $\varepsilon, \mu, \sigma$ being respectively the electric permittivity, magnetic permeability and the conductivity of the propagation medium and $\mathbf{n}$ the outward normal to $\partial \Omega$.

A family of Schwarz methods for (1) with a possibly non-overlapping decomposition of the domain $\Omega$ into $\Omega_{1}$ and $\Omega_{2}$, with interfaces $\Gamma_{12}:=\partial \Omega_{1} \cap \Omega_{2}$ and $\Gamma_{21}:=\partial \Omega_{2} \cap \Omega_{1}$, is given by

$$
\begin{array}{rlrl}
-i \omega \varepsilon \mathbf{E}^{1, n}+\operatorname{curl} \mathbf{H}^{1, n}-\sigma \mathbf{E}^{1, n} & =\mathbf{J} & & \text { in } \Omega_{1}, \\
i \omega \mu \mathbf{H}^{1, n}+\operatorname{curl} \mathbf{E}^{1, n} & =\mathbf{0} & & \text { in } \Omega_{1}, \\
\left(\mathscr{B}_{\mathbf{n}_{1}}+\mathscr{S}_{1} \mathscr{B}_{\mathbf{n}_{2}}\right)\left(\mathbf{E}^{1, n}, \mathbf{H}^{1, n}\right) & =\left(\mathscr{B}_{\mathbf{n}_{1}}+\mathscr{S}_{1} \mathscr{B}_{\mathbf{n}_{2}}\right)\left(\mathbf{E}^{2, n-1}, \mathbf{H}^{2, n-1}\right) & \text { on } \Gamma_{12}, \\
-i \omega \varepsilon \mathbf{E}^{2, n}+\operatorname{curl} \mathbf{H}^{2, n}-\sigma \mathbf{E}^{2, n} & =\mathbf{J} & & \text { in } \Omega_{2}, \\
i \omega \mu \mathbf{H}^{2, n}+\operatorname{curl} \mathbf{E}^{2, n} & =\mathbf{0} & & \text { in } \Omega_{2}, \\
\left(\mathscr{B}_{\mathbf{n}_{2}}+\mathscr{S}_{2} \mathscr{B}_{\mathbf{n}_{1}}\right)\left(\mathbf{E}^{2, n}, \mathbf{H}^{2, n}\right) & =\left(\mathscr{B}_{\mathbf{n}_{2}}+\mathscr{S}_{2} \mathscr{B}_{\mathbf{n}_{1}}\right)\left(\mathbf{E}^{1, n-1}, \mathbf{H}^{1, n-1}\right) & \text { on } \Gamma_{21},
\end{array}
$$

where $\mathscr{S}_{j}, j=1,2$ are tangential operators. For the case of constant coefficients and the domain $\Omega=\mathbb{R}^{2}$, with the Silver-Müller radiation condition $\lim _{r \rightarrow \infty} r(\mathbf{H} \times \mathbf{n}-\mathbf{E})=$ 0 and the two subdomains $\Omega_{1}=(0, \infty) \times \mathbb{R}, \Omega_{2}=(-\infty, L) \times \mathbb{R}, L \geq 0$, the following convergence result was obtained in [4] using Fourier analysis:

Theorem 1. For $\sigma>0$, if $\mathscr{S}_{j}, j=1,2$ have the constant Fourier symbol

$$
\sigma_{j}=\mathscr{F}\left(\mathscr{S}_{j}\right)=-\frac{s-i \tilde{\omega}}{s+i \tilde{\omega}}, \quad \tilde{\omega}=\omega \sqrt{\varepsilon \mu}, \quad s \in \mathbb{C}
$$

then the optimized Schwarz method (2), has the convergence factor

$$
\rho(k, \tilde{\omega}, Z, \sigma, L, s)=\left|\left(\frac{\sqrt{k^{2}-\tilde{\omega}^{2}+i \tilde{\omega} \sigma Z}-s}{\sqrt{k^{2}-\tilde{\omega}^{2}+i \tilde{\omega} \sigma Z}+s}\right) e^{-\sqrt{k^{2}-\tilde{\omega}^{2}+i \tilde{\omega} \sigma Z} L}\right| .
$$

In order to obtain the most efficient algorithm, we choose $\sigma_{j}, j=1,2$ such that $\rho$ is minimal over the range of numerical frequencies $k \in K=\left[k_{\min }, k_{\max }\right]$, e.g. $k_{\min }=0$ and $k_{\max }=\frac{C}{h}$ with $h$ the mesh size and $C$ a constant. We look for $s$ of the form $s=p+i q$, such that $(p, q)$ is solution of the min-max problem

$$
\left.\rho^{*}:=\min _{p, q \geq 0}\left(\max _{k \in K} \rho(k, \tilde{\omega}, Z, \sigma, L, p+i q)\right)\right) .
$$


In [4] we have solved this min-max problem for the case $p=q$ without overlap, and we have obtained the following result:

Theorem 2. For $\sigma>0$ and $L=0$, the solution of the min-max problem (5) with $p=q$ is for $h$ small given by

$$
p^{*}=\frac{(\omega \sigma \mu)^{\frac{1}{4}} \sqrt{C}}{2^{\frac{1}{4}} \sqrt{h}} \quad \text { and } \quad \rho_{1}^{*}=1-\frac{2^{\frac{3}{4}}(\omega \sigma \mu)^{\frac{1}{4}} \sqrt{h}}{\sqrt{C}}+O(h) .
$$

For the overlapping case, we obtained in [8]:

Theorem 3. For $\sigma>0$ and $L=h$, a local minimum of the min-max problem (5) with $p=q$ is for $h$ small given by

$$
p^{*}=\frac{(2 \omega \sigma \mu)^{\frac{1}{3}}}{2 h^{\frac{1}{3}}} \quad \text { and } \quad \rho_{1 L}^{*}=1-2^{\frac{7}{6}}(\omega \sigma \mu)^{\frac{1}{6}} h^{\frac{1}{3}}+O\left(h^{\frac{2}{3}}\right) .
$$

\section{Analysis of the two parameter family of transmission conditions}

As before, we set $k_{\min }=0, k_{\max }=\frac{C}{h}$ and denote by $\left(p^{*}, q^{*}\right)$ a local minimum of (5). We first consider the non-overlapping case.

Theorem 4. For $\sigma>0$ and $L=0$, a local minimum $\left(p^{*}, q^{*}\right)$ of $(5)$ is for $h$ small given by

$$
p^{*}=\frac{3^{\frac{3}{8}}(\omega \sigma \mu)^{\frac{1}{4}} \sqrt{C}}{2^{\frac{3}{4}} \sqrt{h}}, q^{*}=\frac{3^{\frac{7}{8}}(2 \omega \sigma \mu)^{\frac{1}{4}} \sqrt{C}}{6 \sqrt{h}}, \rho_{2}^{*}=1-\frac{3^{\frac{3}{8}}(2 \omega \sigma \mu)^{\frac{1}{4}} \sqrt{h}}{\sqrt{C}}+O(h) .
$$

Proof. By solving the min-max problem (5) numerically for different parameter values and different mesh sizes $h$, we observe that the solution of (5) equioscillates once, i.e. $\left(p^{*}, q^{*}\right)$ is solution of

$$
\rho\left(\bar{k}, \tilde{\omega}, \sigma, Z, 0, p^{*}+i q^{*}\right)=\rho\left(k_{\max }, \tilde{\omega}, \sigma, Z, 0, p^{*}+i q^{*}\right),
$$

where $\bar{k}$ is an interior local maximum of $\rho$. We also observe the asymptotic behavior

$$
\bar{k} \sim \bar{C}, \quad p^{*} \sim C_{p} h^{-\frac{1}{2}}, \quad q^{*} \sim C_{q} h^{-\frac{1}{2}} .
$$

In order to determine the constants $\bar{C}, C_{p}$ and $C_{q}$, it is necessary to have three equations. The first is (9), the second describes the interior local maximum of $\rho$ in $k$,

$$
\left.\frac{\partial \rho}{\partial k}\left(\bar{k}, \tilde{\omega}, \sigma, Z, 0, p^{*}+i q^{*}\right)\right)=0
$$


and the third is the necessary condition for a local minimum of the min-max problem,

$$
\begin{aligned}
& \frac{d \rho}{d q}\left(k_{\max }, \tilde{\omega}, \sigma, Z, 0, p^{*}+i q^{*}\right)= \\
& \quad \frac{\partial \rho}{\partial q}\left(k_{\max }, \tilde{\omega}, \sigma, Z, 0, p^{*}+i q^{*}\right)+\frac{\partial \rho}{\partial p}\left(k_{\max }, \tilde{\omega}, \sigma, Z, 0, p^{*}+i q^{*}\right) \frac{\partial p}{\partial q}=0 .
\end{aligned}
$$

Since $\frac{d \rho}{d q}\left(k_{\max }, \tilde{\omega}, \sigma, Z, 0, p^{*}+i q^{*}\right)=\frac{d \rho}{d q}\left(\bar{k}, \tilde{\omega}, \sigma, Z, 0, p^{*}+i q^{*}\right)$ a similar expansion together with the previous one, gives

$$
\frac{\partial p}{\partial q}=-\frac{\frac{\partial \rho}{\partial q}\left(k_{\max }, \tilde{\omega}, \sigma, Z, 0, p^{*}+i q^{*}\right)-\frac{\partial \rho}{\partial q}\left(\bar{k}, \tilde{\omega}, \sigma, Z, 0, p^{*}+i q^{*}\right)}{\frac{\partial \rho}{\partial p}\left(k_{\max }, \tilde{\omega}, \sigma, Z, 0, p^{*}+i q^{*}\right)-\frac{\partial \rho}{\partial p}\left(\bar{k}, \tilde{\omega}, \sigma, Z, 0, p^{*}+i q^{*}\right)},
$$

and thus asymptotically, the three equations lead to the system

$$
\begin{aligned}
\left(\sqrt{A_{1}}+\bar{C}^{2}-\tilde{\omega}^{2}\right)\left(A C_{p}+B C_{q}\right)-2 \sqrt{A_{1}} B C_{q} & =0, \\
2 C_{p}\left(C_{p}^{2}+C_{q}^{2}\right)-C\left(B C_{p}+A C_{q}\right) & =0, \\
A\left(C_{q}^{2}-C_{p}^{2}\right)+2 C_{p} C_{q} B & =0,
\end{aligned}
$$

where $A=\sqrt{2 \sqrt{A_{1}}-A_{2}}, B=\sqrt{2 \sqrt{A_{1}}+A_{2}}, A_{1}=\bar{C}^{4}-2(\bar{C} \tilde{\omega})^{2}+\tilde{\omega}^{4}+(\tilde{\omega} \sigma Z)^{2}$ and $A_{2}=2\left(\bar{C}^{2}-\tilde{\omega}^{2}\right)$. The solution of this system is

$$
\bar{C}=\frac{\sqrt{\tilde{\omega}(-Z \sigma \sqrt{3}+3 \tilde{\omega})}}{\sqrt{3}}, \quad C_{p}=\frac{3^{\frac{3}{8}}(\tilde{\omega} \sigma Z)^{\frac{1}{4}} \sqrt{C}}{2^{\frac{3}{4}}}, \quad C_{q}=\frac{3^{\frac{7}{8}}(2 \tilde{\omega} \sigma Z)^{\frac{1}{4}} \sqrt{C}}{6},
$$

from which (8) follows. It remains to show that $\left(p^{*}, q^{*}\right)$ is a local minimum, i.e. for any variation $(\delta p, \delta q)$ and $k \in\left\{\bar{k}, k_{\max }\right\}$, we must have

$$
\rho\left(k, \tilde{\omega}, \sigma, Z, 0, p^{*}+\delta p+i\left(q^{*}+\delta q\right)\right) \geq \rho\left(k, \tilde{\omega}, \sigma, Z, 0, p^{*}+i q^{*}\right) .
$$

By the Taylor formula, it suffices to prove that there is no variation $(\delta p, \delta q)$ such that for $k \in\left\{\bar{k}, k_{\max }\right\}$

$$
\delta p \frac{\partial \rho}{\partial p}\left(k, \tilde{\omega}, \sigma, Z, 0, p^{*}+i q^{*}\right)+\delta q \frac{\partial \rho}{\partial q}\left(k, \tilde{\omega}, \sigma, Z, 0, p^{*}+i q^{*}\right)<0 .
$$

We prove this by contradiction, and it is necessary to obtain the next higher order terms in the expansions of $p^{*}, q^{*}$ and $\bar{k}$. After a lengthy computation, we find that asymptotically

$$
\bar{k} \sim \bar{C}+\tilde{C} h, \quad p^{*} \sim C_{p} h^{-\frac{1}{2}}+\tilde{C}_{p} h^{\frac{3}{2}}, \quad q^{*} \sim C_{q} h^{-\frac{1}{2}}+\tilde{C}_{q} h^{\frac{1}{2}} .
$$

The computation of these new three constants allows us to obtain the partial derivatives of $\rho$ 


$$
\begin{aligned}
& \frac{\partial \rho}{\partial p}(\bar{k}) \sim \frac{2}{C} h, \frac{\partial \rho}{\partial q}(\bar{k}) \sim-\frac{3^{\frac{1}{4}}(2 \omega \sigma \mu)^{\frac{1}{2}}}{C^{2}} h^{2}, \\
& \frac{\partial \rho}{\partial p}\left(k_{\max }\right) \sim-\frac{2}{C} h, \frac{\partial \rho}{\partial q}\left(k_{\max }\right) \sim \frac{3^{\frac{1}{4}}(2 \omega \sigma \mu)^{\frac{1}{2}}}{C^{2}} h^{2} .
\end{aligned}
$$

Introducing these results into (10), we get $\delta p \frac{2}{C} h-\delta q \frac{3^{\frac{1}{4}}(2 \omega \sigma \mu)^{\frac{1}{2}}}{C^{2}} h^{2}<0$ and $-\delta p \frac{2}{C} h+$ $\delta q \frac{3^{\frac{1}{4}}(2 \omega \sigma \mu)^{\frac{1}{2}}}{C^{2}} h^{2}<0$, clearly a contradiction, and thus $\left(p^{*}, q^{*}\right)$ is a local minimum.

We see that for $h$ small, both the one parameter and two parameter transmission conditions can be written as $\rho_{1}^{*}=1-\alpha_{1} \sqrt{h}+O(h)$ and $\rho_{2}^{*}=1-\alpha_{2} \sqrt{h}+O(h)$. The ratio $\frac{\alpha_{2}}{\alpha_{1}}$ is equal to $3^{\frac{3}{8}} / \sqrt{2} \approx 1.067$, which shows that the convergence factors are almost equal. Hence the hypothesis $p=q$, used in [4] to simplify the analysis, is justified.

We treat now the overlapping case of (5), with an overlap of one mesh size.

Theorem 5. For $\sigma>0$ and $L=h$, a local minimum $\left(p^{*}, q^{*}\right)$ of (5) is for $h$ small given by

$$
p^{*}=\frac{3^{\frac{1}{2}}(\omega \sigma \mu)^{\frac{1}{3}}}{2^{\frac{4}{3}} h^{\frac{1}{3}}}, \quad q^{*}=\frac{(\omega \sigma \mu)^{\frac{1}{3}}}{2^{\frac{4}{3}} h^{\frac{1}{3}}}, \quad \rho_{2 L}^{*}=1-2^{\frac{5}{6}} 3^{\frac{3}{8}}(\omega \sigma \mu)^{\frac{1}{6}} h^{\frac{1}{3}}+O\left(h^{\frac{2}{3}}\right) .
$$

Proof. As in the proof of Theorem 4, we first observe numerically that the solution of (5) equioscillates once, i.e. $\left(p^{*}, q^{*}\right)$ is solution of

$$
\rho\left(\bar{k}_{1}, \tilde{\omega}, \sigma, Z, h, p^{*}+i q^{*}\right)=\rho\left(\bar{k}_{2}, \tilde{\omega}, \sigma, Z, h, p^{*}+i q^{*}\right),
$$

where $\bar{k}_{1}$ and $\bar{k}_{2}$ are interior local maxima of $\rho$, and we obtain asymptotically for $h$ small

$$
\bar{k}_{1} \sim C_{b_{1}}, \bar{k}_{2} \sim C_{b_{2}} h^{-\frac{2}{3}}, p^{*} \sim C_{p} h^{-\frac{1}{3}} \text { and } q^{*} \sim C_{q} h^{-\frac{1}{3}} .
$$

It remains to find $C_{b_{1}}, C_{b_{2}}, C_{p}$ and $C_{q}$. Proceeding as before, we obtain four equations from the necessary conditions of a minimum, with solution

$$
C_{p}=\frac{3^{\frac{1}{2}}(2 \omega \sigma \mu)^{\frac{1}{2}}}{2}, C_{q}=\frac{C_{p}}{\sqrt{3}}, C_{b_{1}}=\frac{\sqrt{\tilde{\omega}(-Z \sigma \sqrt{3}+3 \tilde{\omega})}}{\sqrt{3}}, C_{b_{2}}=\sqrt{2 C_{p}},
$$

which leads to (11). To prove that $\left(p^{*}, q^{*}\right)$ is a local minimum, proceeding as before, we obtain after a lengthy computation the higher order expansion

$$
\bar{k}_{1} \sim C_{b_{1}}+\tilde{C}_{b_{1}} h^{\frac{2}{3}}, \bar{k}_{2} \sim C_{b_{2}} h^{-\frac{2}{3}}+\tilde{C}_{b_{2}}, p^{*} \sim C_{p} h^{-\frac{1}{3}}+\tilde{C}_{p} h^{\frac{1}{3}}, q^{*} \sim C_{q} h^{-\frac{1}{3}}+\tilde{C}_{q} h^{\frac{1}{3}} .
$$

The computation of these four new constants allows us then to obtain the partial derivatives of $\rho$, 


$$
\begin{aligned}
& \frac{\partial \rho}{\partial p}\left(\bar{k}_{1}\right) \sim \frac{8 \cdot 2^{\frac{1}{6}} h^{\frac{2}{3}}}{3^{\frac{1}{4}}(\omega \sigma \mu)^{\frac{1}{6}}}, \frac{\partial \rho}{\partial q}\left(\bar{k}_{1}\right) \sim-\frac{2 \cdot 2^{\frac{5}{6}}(\omega \sigma \mu)^{\frac{1}{6}} h^{\frac{4}{3}}}{3^{\frac{1}{4}}}, \\
& \frac{\partial \rho}{\partial p}\left(\bar{k}_{2}\right) \sim-\frac{4 \cdot 2^{\frac{1}{6}} h^{\frac{2}{3}}}{3^{\frac{1}{4}}(\omega \sigma \mu)^{\frac{1}{6}}}, \frac{\partial \rho}{\partial q}\left(\bar{k}_{2}\right) \sim \frac{2^{\frac{5}{6}}(\omega \sigma \mu)^{\frac{1}{6}} h^{\frac{4}{3}}}{3^{\frac{1}{4}}} .
\end{aligned}
$$

In order to reach a contradiction, we assume again there exists, by the Taylor theorem, a variation $(\delta p, \delta q)$ such that $\delta p \frac{\partial \rho}{\partial p}\left(k, \tilde{\omega}, \sigma, Z, h, p^{*}+i q^{*}\right)+\delta q \frac{\partial \rho}{\partial q}\left(k, \tilde{\omega}, \sigma, Z, h, p^{*}+\right.$ $\left.i q^{*}\right)<0$, for $k \in\left\{\bar{k}_{1}, k_{2}\right\}$. Using (12), we get $8 \frac{2^{\frac{1}{6}} h^{\frac{2}{3}}}{3^{\frac{1}{4}}(\omega \sigma \mu)^{\frac{1}{6}}} \delta p-2 \frac{2^{\frac{5}{6}}(\omega \sigma \mu)^{\frac{1}{6}} h^{\frac{4}{3}}}{3^{\frac{1}{4}}} \delta q<0$ and $-4 \frac{2^{\frac{1}{6}} h^{\frac{2}{3}}}{3^{\frac{1}{4}}(\omega \sigma \mu)^{\frac{1}{6}}} \delta p+\frac{2^{\frac{5}{6}}(\omega \sigma \mu)^{\frac{1}{6}} h^{\frac{4}{3}}}{3^{\frac{1}{4}}} \delta q<0$, clearly a contradiction, and thus $\left(p^{*}, q^{*}\right)$ is a local minimum.

We also observe in this case that for $h$ small, both convergence factors can be written as $\rho_{1 L}^{*}=1-\alpha_{1 L} h^{\frac{1}{3}}+O\left(h^{\frac{2}{3}}\right)$ and $\rho_{2 L}^{*}=1-\alpha_{2 L} h^{\frac{1}{3}}+O\left(h^{\frac{2}{3}}\right)$, and the ratio $\frac{\alpha_{2 L}}{\alpha_{1 L}}$ is equal to $3^{\frac{1}{4}} / 2^{\frac{1}{3}} \approx 1.044$, hence both convergence factors are almost equal. We show an example of these convergence factors in Figure 1.
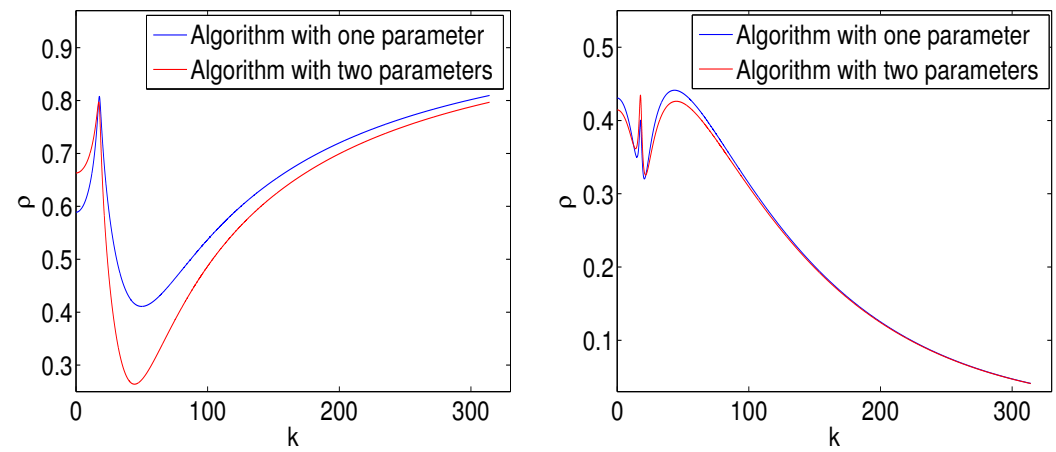

Fig. 1 Convergence factor comparison of algorithms with one and two parameters for $\omega=2 \pi$, $\sigma=2$ and $\mu=\varepsilon=1$, for the non-overlapping case, $L=0$, on the left, and the overlapping case, $L=h=\frac{1}{100}$, on the right

\section{Numerical results}

We present now a numerical test in order to compare the performance of both the one and two parameter algorithms. We compute the propagation of a plane wave in a heterogeneous medium. The domain is $\Omega=(-1,1)^{2}$. The relative permittivity 
and the conductivity of the background media is $\varepsilon_{1}=1.0$ and $\sigma_{1}=1.8$, while that of the square material inclusion is $\varepsilon_{2}=8.0$ and $\sigma_{2}=7.5$, see the left picture of Fig. 2 . The magnetic permeability $\mu$ is constant in $\Omega$ and we impose on the boundary an incident field $\left(H_{x}^{i n c}, H_{y}^{i n c}, E_{z}^{i n c}\right)$. The domain $\Omega$ is decomposed into two subdomains $\Omega_{1}=(-1, L) \times(-1,1)$ and $\Omega_{2}=(0,1) \times(-1,1) ; L$ is the overlapping size and is equal to the mesh size. We use, in each subdomain, a discontinuous Galerkin method (DG) with a uniform polynomial approximation of order one, two and three, denoted by $D G-P 1, D G-P 2$ and $D G-P 3$, see [5]. The results are shown in Fig. 3, and are in good agreement with our analytical results.
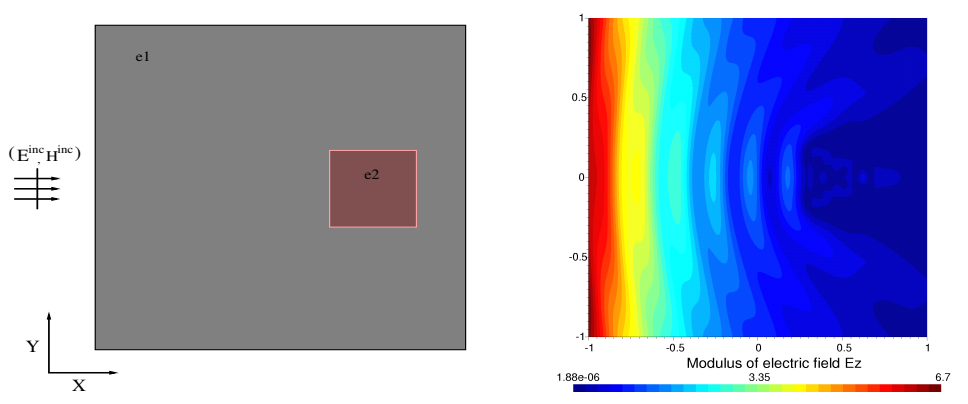

Fig. 2 Configuration of our test problem on the left, and the numerical solution on the right
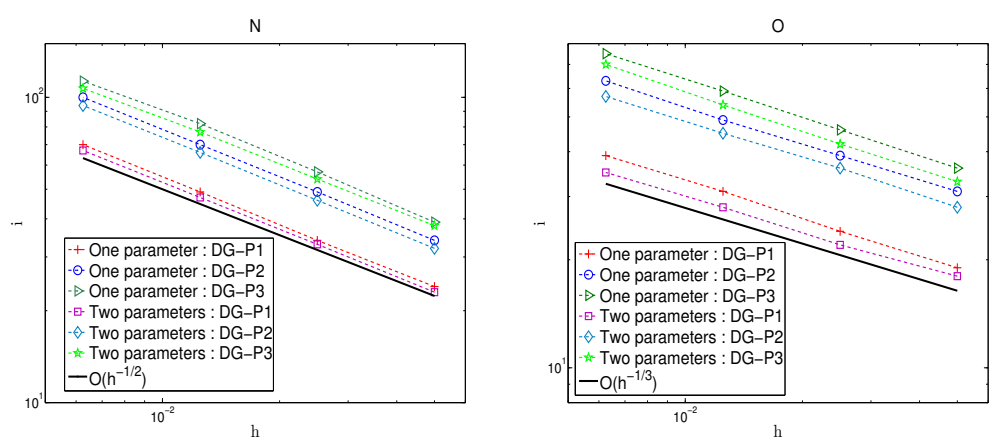

Fig. 3 Number of iterations against the mesh size $h$, to attain a relative residual reduction of $10^{-8}$ 


\section{Conclusion}

We compared in this paper a one and a two parameter family of transmission conditions for optimized Schwarz methods applied to Maxwell's equations. Our asymptotic analysis reveals that the addition of a second parameter does not lead to a significant improvement of the algorithm, and it is therefore justified to consider only the simpler case of a one parameter family of transmission conditions. These results are also confirmed by our numerical experiments.

\section{References}

\section{References}

[1] A. Alonso-Rodriguez and L. Gerardo-Giorda. New nonoverlapping domain decomposition methods for the harmonic Maxwell system. SIAM J. Sci. Comput., 28(1):102-122, 2006.

[2] P. Chevalier and F. Nataf. An OO2 (Optimized Order 2) method for the Helmholtz and Maxwell equations. In 10th International Conference on Domain Decomposition Methods in Science and in Engineering, pages 400-407, Boulder, Colorado, USA, 1997. AMS.

[3] P. Collino, G. Delbue, P. Joly, and A. Piacentini. A new interface condition in the non-overlapping domain decomposition for the Maxwell equations. Comput. Methods Appl. Mech. Engrg., 148:195-207, 1997.

[4] V. Dolean, M. El Bouajaji, M. Gander, and S. Lanteri. Optimized Schwarz methods for Maxwell's equations with non-zero electric conductivity. In Domain Decomposition Methods in Science and Engineering XIX, LNCSE, Vol. 78, pp 269-276, 2011.

[5] V. Dolean, M. El Bouajaji, M. Gander, S. Lanteri, and R. Perrussel. Domain decomposition methods for electromagnetic wave propagation problems in heterogeneous media and complex domains. In Domain Decomposition Methods in Science and Engineering XIX,LNCSE, Vol. 78, pp 15-26, 2011.

[6] V. Dolean, L. Gerardo-Giorda, and M. J. Gander. Optimized Schwarz methods for Maxwell equations. SIAM J. Scient. Comp., 31(3):2193-2213, 2009.

[7] V. Dolean, S. Lanteri, and R. Perrussel. Optimized Schwarz algorithms for solving time-harmonic Maxwell's equations discretized by a discontinuous Galerkin method. IEEE. Trans. Magn., 44(6):954-957, 2008.

[8] V. Dolean, M. El Bouajaji, M. J. Gander, S. Lanteri, Optimized Schwarz methods for the time-harmonic Maxwell equations with damping, SIAM J. Sci. Comput., Vol 34., No. 4, pp. 2048-2071, 2012.

[9] Zhen Peng and Jin-Fa Lee. Non-conformal domain decomposition method with second-order transmission conditions for time-harmonic electromagnetics. J. Comput. Phys., 229(16):5615-5629, 2010. 